\title{
Operational definition of child assent for research
}

Alan R. Tait, Michael E. Geisser

\section{Source}

Alan R. Tait, Michael E. Geisser. (2017). Development of a consensus operational

definition of child assent for research. BMC Med Ethics, vol. 18 (1). doi:10.1186/s12910-

017-0199-4.

Children who lack the legal authority to provide informed consent per state laws should provide their assent to participate in a research study unless they either lack the cognitive ability, their clinical condition precludes their ability to communicate a choice, or the research holds out the prospect of direct benefit that is only available in the context of the research. Assent is an interactive process between a researcher and child participant involving disclosure of cognitively and emotionally appropriate information regarding, at minimum, why the child is being asked to participate, a description of the procedures and how the child might experience them, and an understanding that participation in the study is voluntary. Children should understand that they can decline participation or withdraw from the study at any time. Assent requires that the child explicitly affirms his or her agreement to participate in a manner that reflects their age-appropriate understanding and that is free of undue influence or coercion. In the absence of an explicit agreement, mere failure of the child to object cannot be construed as assent. 